\title{
Occupational exposures and the risk of COPD: dusty trades revisited
}

\author{
P D Blanc, ${ }^{1}$ C Iribarren, ${ }^{2}$ L Trupin, ${ }^{3}$ G Earnest, ${ }^{1}$ P P Katz, ${ }^{3}$ J Balmes, ${ }^{1}$ S Sidney, ${ }^{2}$ \\ M D Eisner ${ }^{1,2}$
}

${ }^{1}$ Division of Occupational and Environmental Medicine \& Division of Pulmonary and Critical Care Medicine, Department of Medicine University of California, San Francisco, USA; ${ }^{2}$ Division of Research, Kaiser Permanente, Oakland, California, USA;

${ }^{3}$ Institute for Health Policy

Studies, Department of

Medicine, University of

California, San Francisco, USA

Correspondence to:

Dr M D Eisner, University of

California, San Francisco, 350

Parnassus Avenue, Ste 609, San

Francisco, CA 94117, USA;

mark.eisner@ucsf.edu

Received 20 March 2008 Accepted 14 July 2008 Accepted for Online First 4 August 2008

\begin{abstract}
Background: The contribution of occupational exposures to chronic obstructive pulmonary disease (COPD) and, in particular, their potential interaction with cigarette smoking remains underappreciated.

Methods: Data from the FLOW study of 1202 subjects with COPD (of which 742 had disease classified as stage II or above by Global Obstructive Lung Disease (GOLD) criteria) and 302 referent subjects matched by age, sex and race recruited from a large managed care organisation were analysed. Occupational exposures were assessed using two methods: self-reported exposure to vapours, gas, dust or fumes on the longest held job (VGDF) and a job exposure matrix (JEM) for probability of exposure based on occupation. Multivariate analysis was used to control for age, sex, race and smoking history. The odds ratio (OR) and adjusted population attributable fraction (PAF) associated with occupational exposure were calculated.
\end{abstract}

Results: VGDF exposure was associated with an increased risk of COPD (OR 2.11; 95\% Cl 1.59 to 2.82) and a PAF of $31 \%(95 \% \mathrm{Cl} 22 \%$ to $39 \%)$. The risk associated with high probability of workplace exposure by JEM was similar (OR 2.27; 95\% Cl 1.46 to 3.52), although the PAF was lower (13\%; $95 \% \mathrm{Cl} 8 \%$ to $18 \%)$. These estimates were not substantively different when the analysis was limited to COPD GOLD stage II or above. Joint exposure to both smoking and occupational factors markedly increased the risk of COPD (OR 14.1; 95\% Cl 9.33 to 21.2)

Conclusions: Workplace exposures are strongly associated with an increased risk of COPD. On a population level, prevention of both smoking and occupational exposure, and especially both together, is needed to prevent the global burden of disease.

Occupational factors are believed to contribute to the population burden of chronic obstructive pulmonary disease (COPD). The American Thoracic Society conducted a systematic epidemiological review and concluded that approximately $15 \%$ of COPD may be attributable to workplace exposures. ${ }^{1}$ A more recent follow-up review provided similar estimates. ${ }^{2}$ Despite this research, the role of the workplace in COPD causation is not widely appreciated, especially the combined effects of "dusty trades" and cigarette smoking.

Cigarette smoking is the dominant risk factor for COPD and must be considered in any analysis of occupational exposures. Previous estimates of the population attributable fraction (PAF) for occupational exposures and COPD risk have statistically adjusted for smoking. ${ }^{12}$ Nonetheless, non-smokers are at risk for developing COPD. Recent analyses have estimated that the PAF for occupational exposures among non-smokers ranges from 11\% to $53 \% .^{3-7}$ The combined effects of smoking and occupational exposures, however, have not been well characterised, even though delineating these joint impacts could have important ramifications for the prevention and clinical management of COPD.

In a previous national survey-based study of adults in the USA we estimated that the PAF for occupational exposure and COPD was $9-31 \%{ }^{8} \mathrm{We}$ also observed a sharp step-up in COPD risk associated with the combination of smoking and occupational exposure. Two subsequent studies also observed a potential interaction between smoking and occupation for chronic bronchitis alone or chronic bronchitis plus airflow obstruction. ${ }^{9}{ }^{10}$ In the current study we elucidated the separate and combined impact of smoking and occupation on the risk of COPD in individuals recruited from a large US managed care organisation who underwent pulmonary function testing.

\section{METHODS}

\section{Overview}

The current study is a matched case-referent analysis nested within the Function, Living, Outcomes and Work (FLOW) study of COPD, which is an ongoing prospective cohort study of adult members of an integrated healthcare delivery system (closed-panel managed care organisation) with a physician's diagnosis of COPD and a matched referent group without COPD. The long-term goal is to determine what factors are responsible for the development of disability in COPD. At baseline assessment we conducted structured telephone interviews to ascertain COPD status, health status, self-reported functional limitations and sociodemographic characteristics. Subjects then underwent a research clinic visit that included spirometry and other physical assessments.

The study was approved by the University of California, San Francisco Committee on Human Research and the Kaiser Foundation Research Institute's institutional review board and all participants provided written informed consent.

\section{COPD subject eligibility}

We studied adult members of Kaiser Permanente Medical Care Program (KPMCP), the largest nonprofit managed care organisation in the USA. In northern California the KPMCP provides the full 
spectrum of primary-to-tertiary care to approximately 3.1 million members (25-30\% of the regional population). ${ }^{11}$ The demographic characteristics of KPMCP members are similar to the overall northern California population, except for the extremes of income distribution. ${ }^{12}$

We identified all adult KPMCP members who were recently treated for COPD using a previously described approach. ${ }^{13}$ The age range was restricted to 40-65 years because a key study outcome includes work disability. ${ }^{14}$ Using KPMCP computerised databases, we identified all subjects who met each of two criteria: one based on healthcare utilisation and the second based on medication prescribing. The healthcare utilisation criterion was one or more ambulatory visits, emergency department visits or hospitalisations with a principal International Classification of Disease (ICD-9) diagnosis code for COPD (chronic bronchitis (491), emphysema (492) or COPD (496)) during a recent 12 -month time period. The medication criterion was two or more prescriptions for a COPD-related medication during a 12-month window beginning 6 months before the index utilisation date and ending 6 months after the index date. The criterion medications included inhaled anticholinergic medications, inhaled $\beta$ agonists, inhaled corticosteroids and theophylline. Based on medical record review, we have shown that this algorithm is a valid method for identifying adults with COPD. ${ }^{14}$ To facilitate attendance at the research clinic we restricted the sample to persons living within a 30-mile geographical radius of the research clinic where the study examinations took place.

Individuals identified by the algorithm who were no longer KPMCP members or who had moved away were considered ineligible for study. The primary care physicians for all patients were contacted and given the opportunity to decline contact of any identified patients under their care. Potential subjects were then contacted by a letter describing the study and given an opportunity to decline participation. Those not declining were then contacted by telephone to arrange an interview. At the end of the interview, subjects were invited to participate in the research clinic visit. Those who were found at the time of interview to have severe communication difficulties attributable to advanced dementia or aphasia were excluded.

\section{COPD subject participation}

A total of 5800 subjects were initially identified using the computerised algorithm. Of these, 298 died before they could be recruited into the study and 1011 did not meet study inclusion criteria or were excluded at the time of interview contact, as noted above. The completion rate for structured telephone interviews was 2310 of the remaining eligible group of 4419 (51\%). This is comparable to our earlier cohort study of adult asthma conducted at KPMCP and compares favourably with other survey-based epidemiological studies conducted in the USA. ${ }^{15}{ }^{16}$ Of the 2310 respondents, 112 were not eligible for the clinic visit (8 subsequently died, 10 were no longer members of KPMCP, 85 were physically unable to attend and 9 moved out of the area). Of the 2198 eligible subjects, 1216 completed the research clinic visit (55\% of those interviewed and eligible). Of these, 10 subjects were excluded because they did not meet the GOLD criteria for COPD after interviews and spirometric tests were performed ${ }^{17}$ and a further 4 subjects were excluded from the analysis because they could not perform spirometry owing to previous tracheostomy placement. Ultimately, 1202 subjects with COPD completed both interviews and research clinic visits.
Demographic information was available for interviewed subjects from their structured telephone interviews and noninterviewed subjects from Kaiser computerised databases. Compared with subjects who were eligible but not interviewed, interviewed subjects were slightly older (by 0.7 years on average), more likely to be female (59\% vs $51 \%$ ) and more likely to be white (69\% vs $56 \%$ ). In terms of race-ethnicity, the two largest minority subgroups were slightly over-represented among those who completed interviews compared with those who did not (Black/African American 14\% vs 11\%, Hispanic 9\% vs $4 \%$ ). Most of the differences in race were driven by limitations inherent in the Kaiser computerised databases: the prevalence of unknown race was much higher among those who did not complete interviews ( $17 \%$ vs $0.3 \%$ ).

Compared with subjects who completed interviews but not the clinic visit, clinic visit attendees were similar in age (mean difference 0.3 years), sex (58\% vs $55 \%$ female), and raceethnicity (67\% vs $61 \%$ white). Black or Hispanic subjects were more likely to complete the research clinic visit (17\% completed vs $11 \%$ not completed and $9 \%$ vs $5 \%$, respectively).

\section{Study referent group}

We aimed to recruit 300 referent subjects without COPD. Using recruitment methods similar to those for subjects with COPD, we initially identified 373 referent subjects with no history of COPD who were matched to subjects with COPD by age, sex and race. By design, we subsequently excluded 71 subjects who had evidence of airway obstruction (forced expiratory volume in $1 \mathrm{~s} /$ forced vital capacity $\left.\left(\mathrm{FEV}_{1} / \mathrm{FVC}\right)<0.70\right)$ at the time of research clinic evaluation, leaving 302 referent subjects for the final analysis.

\section{Structured telephone interviews}

All subjects (those with COPD and non-COPD referents) underwent 30-40 min structured telephone interviews facilitated with customised computer-assisted telephone interview software. Interviews ascertained a wide range of data potentially relevant to health status. These included items assessing sociodemographic characteristics, cigarette smoking and occupational histories. As in previous studies, we defined educational attainment as high school or less, some college or college/ graduate degree. ${ }^{18}$ Race-ethnicity was categorised as previously described. ${ }^{18}$

Cigarette smoking was measured using questions developed for the National Health Interview Survey and was defined as current smoking, past smoking or never smoked and, in a secondary analysis, $<20$ or $\geqslant 20$ pack-years smoking. ${ }^{19}$

Open ended items elicited occupation, industry and main job duties for the respondent's longest held job. Occupation was then coded using the US 2000 census codes. ${ }^{20}$ Each subject was asked about self-reported exposures using a questionnaire item used in the baseline European Community Respiratory Health Survey (ECRHS I). This asks respondents whether they were exposed to vapours, gas, dust or fumes (VGDF) on the job. ${ }^{21}$

\section{Job exposure matrix}

In addition to self-reported exposure, we also assessed occupational risk using a job exposure matrix (JEM). This JEM was initially developed in an analysis of respiratory disability and later modified to use in asthma- and COPD-specific versions. ${ }^{82-24}$ The JEM categorises specific occupations as having a low, intermediate or high probability of exposure to materials associated with chronic airway disease. 
The COPD JEM differs from the asthma JEM in several ways. In particular, the COPD matrix classifies occupations likely to involve sensitisers (eg, latex) as low probability for COPD, whereas these exposures are in the high-risk category in the asthma-specific JEM. In addition, occupations with a high likelihood of second hand smoke exposure (eg, waitresses, bartenders) are defined as having a moderate COPD risk; occupations with diesel exhaust exposure (eg, truck and heavy equipment operators) and dusty trades (eg, most construction jobs) are defined as high risk in the COPD-specific JEM.

For this analysis we further adapted the COPD JEM using narrative open text fields of job descriptions to modify classifications otherwise assigned by occupational code alone, a so-called "expert review" step that has been recommended to improve JEM performance. ${ }^{25}$

\section{Assessment of pulmonary function}

To measure pulmonary function we conducted spirometry according to American Thoracic Society (ATS) guidelines. ${ }^{26}{ }^{27}$ We used the EasyOne Frontline spirometer (ndd Medical Technologies, Chelmsford, MA, USA) which is known for its reliability, accuracy and durability and has been widely used in epidemiological research. ${ }^{28}{ }^{29}$ Although no calibration is recommended by the company, we checked the device daily using a 3litre syringe (Hans-Rudolph, Kansas City, MO, USA). During the study period the device was always within a range of $\pm 1 \%$.

To calculate percentage predicted pulmonary function values we used predictive equations derived from NHANES III. ${ }^{30}$ Based on $\mathrm{FEV}_{1}, \mathrm{FEV}_{1} / \mathrm{FVC}$ ratio and respiratory symptoms, the severity of COPD was staged according to the NHLBI/WHO Global Initiative for Chronic Obstructive Lung Disease (GOLD) criteria (stage 0-IV). ${ }^{1731}$ Because research clinic examinations were conducted by trained non-medical personnel, we did not administer bronchodilators for study purposes. However, $90 \%$ of subjects had taken their own short-acting bronchodilator within $4 \mathrm{~h}$ of spirometry or had taken a long-acting bronchodilator earlier in the same day.

\section{Statistical analysis}

Statistical analysis was conducted using SAS software Version 9.1 (SAS Institute, Cary, NC, USA) and Stata 10 (College Park, TX, USA). Bivariate comparisons were carried out using a $t$ test for continuous variables and the $\chi^{2}$ test for categorical variables. Logistic regression analysis was used to examine the association between self-reported occupational VGDF exposure and the risk of COPD, controlling for age, sex, race and smoking history (past smoking and current smoking as indicator variables). Education and income were not included in the analysis because they are determinants and consequences of occupational status, respectively, and are therefore on the causal pathway between occupation and COPD (rather than functioning as confounders). ${ }^{8}$ In additional parallel analyses we substituted JEMassessed occupational risk for self-assessed exposure. We also reanalysed occupational risk in the never smoking stratum of subjects and in the never smokers combined with very minimal smoking histories ( $<5$ pack-years). In another secondary analysis we re-estimated the main models substituting $<20$ pack-years and $\geqslant 20$ pack-years of smoking in place of the exsmoking and current smoking indicator variables. We also reestimated the main models redefining smoking as cumulative pack-years, which had no substantive impact on the results (data not shown).
We calculated the population attributable fraction (also known as the population attributable risk percent or PAR\%) to estimate the proportion of COPD prevalence attributable to occupational exposure (measured by self-report of VGDF and, separately, by JEM) after adjusting for smoking status and other covariates. The adjusted PAF was estimated from the multivariate logistic regression analysis using the method of Greenland and Drescher. ${ }^{32}$

The interaction between smoking status and occupational VGDF exposure was explicitly evaluated. For these analyses, smoking was dichotomised as ever versus never and occupational exposure was defined as exposure to VGDF during the longest held job versus not. Four cells were therefore possible, given each dichotomous combination, with the referent category being never smoking and no occupational exposure. We estimated the joint association between smoking and VGDF as both excess probability of COPD and odds ratio for each smoking-VGDF category, which is modelled after our previous approach. ${ }^{8}$

To evaluate a more severe spectrum of COPD we repeated our key analyses, redefining COPD as an $\mathrm{FEV}_{1} / \mathrm{FVC}$ ratio of $<0.70$ and $\mathrm{FEV}_{1}<80 \%$ predicted (ie, GOLD stage II or greater, consistent with the Burden of Lung Disease (BOLD) Study strategy). ${ }^{33}$ In these analyses we eliminated subjects with less severe disease.

\section{RESULTS}

Demographic characteristics, smoking and pulmonary function Subjects with COPD were similar to referents in terms of age, sex and race ( $>>0.20$, table 1). Those with COPD had significantly lower educational attainment and annual household income $(p<0.001)$. Consistent with the study design, cigarette smoking differed substantially between subjects with COPD and referents ( $87 \%$ vs $48 \%$ ever smokers). Of the 1202 subjects with COPD, $742(42 \%)$ had GOLD stage II or greater disease severity.

\section{Occupational characteristics and workplace exposures}

Lifetime labour force participation (ie, ever worked) did not differ statistically between those with COPD and referents, but persons with COPD were less likely to be currently employed $(p<0.001$, table 2). This pattern was similar for the overall COPD group and those with GOLD stage II and above. As shown in table 2, occupational exposure was significantly more common in COPD, whether it was ascertained by self-report or JEM ( $p<0.0001$ for both). Within the COPD group overall, the majority reported VGDF exposure $(58 \%)$ whereas $32 \%$ were assigned to an intermediate or high exposure probability by JEM classification.

\section{Occupation and the risk for COPD}

Adjusted for smoking and other covariates, self-reported VGDF exposure during the longest held job was associated with double the odds of COPD (OR 2.11; 95\% CI 1.59 to 2.82) and a PAF of $31 \%$ (95\% CI $22 \%$ to $39 \%$ ) (table 3). The risk associated with high probability of exposure by JEM was similar to self-assessed VGDF exposure (OR 2.27; 95\% CI 1.46 to 3.52), although the PAF was lower (13\%; 95\% CI $8 \%$ to 18\%). The lower PAF for JEM-assessed exposure reflects the lower prevalence of exposed COPD cases by this metric. These estimates were not substantively different when the analysis was limited to COPD GOLD stage II or above. 
Table 1 Baseline characteristics of FLOW cohort of subjects with chronic obstructive pulmonary disease (COPD) and referent subjects

\begin{tabular}{|c|c|c|c|}
\hline Characteristic & $\begin{array}{l}\text { COPD } \\
(\mathrm{n}=1202)\end{array}$ & $\begin{array}{l}\text { Referents } \\
(n=302)\end{array}$ & p Value \\
\hline Mean (SD) age (years) & $58.2(6.2)$ & $58.5(6.2)$ & 0.50 \\
\hline Female, n (\%) & $691(57.4 \%)$ & $185(61 \%)$ & 0.23 \\
\hline Race/ethnicity, n (\%) & & & 0.96 \\
\hline White, non-Hispanic & $810(67 \%)$ & $200(66 \%)$ & \\
\hline African-American & $206(17 \%)$ & $57(19 \%)$ & \\
\hline Asian/Pacific Islander & $35(3 \%)$ & $8(3 \%)$ & \\
\hline Hispanic & $111(9 \%)$ & $28(9 \%)$ & \\
\hline Other & $40(3 \%)$ & $9(3 \%)$ & \\
\hline Educational attainment, n (\%) & & & $<0.0001$ \\
\hline High school or less & $352(29 \%)$ & $42(14 \%)$ & \\
\hline Some college & 524 (44\%) & $95(31 \%)$ & \\
\hline College/graduate degree & $326(27 \%)$ & $165(55 \%)$ & \\
\hline Household income, ${ }^{*} \mathrm{n}(\%)$ & & & $<0.0001$ \\
\hline Low $(<\$ 20000)$ & $129(11 \%)$ & $9(3 \%)$ & \\
\hline$\$ 20000-80000$ & $699(58 \%)$ & $137(45 \%)$ & \\
\hline High (>\$80 000) & $276(23 \%)$ & $133(44 \%)$ & \\
\hline Smoking status, n (\%) & & & $<0.0001$ \\
\hline Never smoked & $165(13 \%)$ & $158(52 \%)$ & \\
\hline Current smoker & 393 (33\%) & $12(4 \%)$ & \\
\hline Ex-smoker & $644(54 \%)$ & $132(44 \%)$ & \\
\hline Smoking, n (\%) & & & $<0.001$ \\
\hline None & $162(13 \%)$ & $158(52 \%)$ & \\
\hline$<20$ pack-years & $627(52 \%)$ & $92(30 \%)$ & $0.43 \dagger$ \\
\hline$\geqslant 20$ pack-years & $410(34 \%)$ & $52(17 \%)$ & \\
\hline
\end{tabular}

*98 $(8 \%)$ of the COPD group and $23(8 \%)$ of the referent group declined to state income.

$\dagger$ Comparing proportions of $<20$ vs $\geqslant 20$ pack-years among ever smokers only.

Cigarette smoking was strongly associated with the risk of COPD (table 3). The combined PAF for smoking (current and past) was $74 \%$ for the entire cohort and $81 \%$ for subjects with COPD GOLD stage II and above. Re-estimating the models using smoking duration-intensity (dichotomised as $<20$ and $\geqslant 20$ pack-years) did not substantively alter the occupational risk estimates (data not shown).

Among lifelong never smokers $(n=323)$, VGDF exposure during the longest held job was associated with a greater risk of COPD after controlling for covariates (OR 2.0; 95\% CI 1.28 to 3.18). The estimated PAF in this stratum was $26 \%$ (95\% CI $10 \%$ to $40 \%$ ). Although there was no clear association between the intermediate JEM category and COPD risk (OR 0.79; 95\% CI 0.29 to 2.20 ), the high JEM category appeared to be related to a higher risk of COPD (OR 1.58; 95\% CI 0.72 to 3.50). When the non-smoker stratum was expanded to include both never smokers and also those with minimal cumulative lifetime smoking ( $<5$ pack-years, $\mathrm{n}=618$ ), the high JEM category was more strongly associated with the risk of COPD (OR 2.51; 95\% CI 1.30 to 4.84 and PAF $13 \%$; $95 \%$ CI $6 \%$ to $19 \%$ ).

\section{Joint influence of occupational exposures and smoking}

A step-up in risk associated with combined occupational and smoking exposures was observed (table 4). Those reporting VGDF exposure alone had double the odds of COPD compared with those with neither occupational exposures nor smoking risk (OR 1.98; 95\% CI 1.26 to 3.09). Those with combined exposure had a 14-fold increased risk of COPD, whereas smoking alone was associated with a 7-fold increased risk. A similar magnitude of step-up was evident based on JEM exposure assessment. The excess probability analysis indicates that joint risk for smoking and occupational exposure is additive
Table 2 Employment history and occupational exposures

\begin{tabular}{lccc}
\hline & COPD* & Referent & $\begin{array}{c}\text { p Value for } \\
\text { comparison }\end{array}$ \\
\hline Entire COPD cohort $(n=1202)$ & & & \\
Ever employed & $299(99 \%)$ & $1194(99 \%)$ & 0.55 \\
Currently employed & $595(50 \%)$ & $190(63 \%)$ & $<0.0001$ \\
VGDF exposure during longest held job & $697(58 \%)$ & $117(39 \%)$ & $<0.0001$ \\
JEM exposure probability & & & $<0.0001$ \\
$\quad$ Low & $817(68 \%)$ & $250(83 \%)$ & \\
$\quad$ Intermediate & $107(9 \%)$ & $20(7 \%)$ & \\
$\quad$ High & $278(23 \%)$ & $32(11 \%)$ & \\
& & & \\
GOLD stage II or higher $(n=742)$ & & & \\
Ever employed & $737(99 \%)$ & $1194(99 \%)$ & 0.59 \\
Currently employed & $343(46 \%)$ & $190(63 \%)$ & $<0.0001$ \\
VGDF exposure during longest held job & $442(60 \%)$ & $117(39 \%)$ & $<0.0001$ \\
JEM exposure probability & & & $<0.0001$ \\
$\quad$ Low & $495(67 \%)$ & $250(83 \%)$ & \\
$\quad$ Intermediate & $70(9 \%)$ & $20(7 \%)$ & \\
$\quad$ High & $177(24 \%)$ & $32(11 \%)$ & \\
\hline
\end{tabular}

COPD, chronic obstructive pulmonary disease; GOLD, Global Obstructive Lung Disease initiative; JEM, job exposure matrix for exposure likelihood on longest held job; VGDF, vapours, gas, dust or fume on longest held job by self-report.

*First set of results are for the entire FLOW cohort of COPD subjects vs referents; second set of results (lower portion of table) are for $\geqslant G O L D$ stage II COPD vs referents.

when COPD was defined as GOLD stage II or higher and slightly less than additive in the entire FLOW cohort. Either way, the joint exposure to smoking and occupational exposures was related to a marked increase in the risk of COPD.

Excess probability results indicate that the joint risk for smoking and occupational exposure was additive when COPD was defined as GOLD stage II or higher, whereas it was slightly less than additive in the entire FLOW cohort. For example, in those with COPD GOLD stage II or higher, the joint occurrence of smoking and occupational exposure (excess probability of 0.61) was equal to the sum of smoking alone plus occupation alone (excess probabilities $0.49+0.12=0.61$ ), which is consistent with an additive interaction.

\section{DISCUSSION}

Occupational exposures were found to be strongly associated with the risk of developing COPD. Depending on the workplace exposure metric employed, the PAF ranged between $15 \%$ and $31 \%$ after controlling for smoking and other covariates. Exposure to both smoking and occupational factors portended an especially high risk of COPD. The joint analysis of smoking and workplace exposures implies that elimination of one-but not the other-risk will not be fully effective for reducing the burden of COPD in the population.

Our study advances the field by providing important new evidence that occupational exposures contribute to the overall burden of COPD. The current results are very similar to our previous study which had a different sampling scheme (population-based sampling of the US population vs patients from a managed care organisation) and COPD definition (selfreported diagnosis of chronic bronchitis, emphysema or COPD itself vs GOLD definition based on pulmonary function). ${ }^{8}$ Our results are also consistent with a recent Kaiser Permanente study from north-west USA that identified COPD cases using administrative data and used an exposure assessment method that was a hybrid between the JEM and self-reported exposure metrics used in our study. This study found a 
Table 3 Occupational exposures and the risk of COPD

\begin{tabular}{|c|c|c|c|}
\hline Exposure & Unadjusted OR (95\% CI) & Adjusted OR (95\% CI) & PAF (95\% Cl) \\
\hline \multicolumn{4}{|l|}{ Entire FLOW cohort } \\
\hline VGDF exposure* & 2.18 (1.69 to 2.83 ) & 2.11 (1.59 to 2.82 ) & $31 \%(22 \%$ to $39 \%)$ \\
\hline \multicolumn{4}{|c|}{ JEM exposure probability* } \\
\hline Low (referent) & 1.0 (referent) & 1.0 (referent) & Referent \\
\hline Intermediate & $1.64(1.00$ to 2.67$)$ & 1.27 (0.74 to 2.19$)$ & $2 \%(-2 \%$ to $6 \%)$ \\
\hline High & 2.66 (1.80 to 3.94$)$ & 2.27 (1.46 to 3.52$)$ & $13 \%(8 \%$ to $18 \%)$ \\
\hline \multicolumn{4}{|l|}{ Cigarette smoking } \\
\hline Never (referent) & 1.0 & 1.0 & Referent \\
\hline Current smoker & 31 (17 to 58$)$ & 31 (17 to 58$)$ & $32 \%(30 \%$ to $33 \%)$ \\
\hline Past smoker & 4.67 (3.50 to 6.23 ) & 4.52 (3.35 to 6.09$)$ & $42 \%(37 \%$ to $46 \%)$ \\
\hline \multicolumn{4}{|c|}{ GOLD stage // or higher } \\
\hline VGDF exposure* & $2.33(1.77$ to 3.06$)$ & $2.13(1.55$ to 2.93$)$ & $31 \%(21 \%$ to $41 \%)$ \\
\hline \multicolumn{4}{|c|}{ JEM exposure probability* } \\
\hline Low (referent) & 1.0 (referent) & 1.0 (referent) & Referent \\
\hline Intermediate & 1.77 (1.05 to 2.97$)$ & 1.58 (0.88 to 2.84$)$ & $3 \%(-1 \%$ to $8 \%)$ \\
\hline High & 2.79 (1.86 to 4.19$)$ & 2.33 (1.45 to 3.72 ) & $14 \%$ (8\% to $20 \%$ ) \\
\hline \multicolumn{4}{|l|}{ Cigarette smoking } \\
\hline Never (referent) & 1.0 & 1.0 & Referent \\
\hline Current smoker & 45 (24 to 86$)$ & 46 (24 to 88$)$ & $35 \%(32 \%$ to $37 \%)$ \\
\hline Past smoker & 7.05 (5.02 to 9.91$)$ & 6.27 (4.42 to 8.90$)$ & $46 \%(41 \%$ to $50 \%)$ \\
\hline \multicolumn{4}{|c|}{$\begin{array}{l}\text { COPD, chronic obstructive pulmonary disease; GOLD, Global Obstructive Lung Disease initiative; JEM, job exposure matrix for } \\
\text { exposure likelihood on longest held job; PAF, population attributable fraction based on multivariate analysis; VGDF, vapours, gas, } \\
\text { dust or fume on longest held job by self-report. } \\
\text { *Unadjusted results are from logistic regression models with only that set of variables; adjusted results are from two separate } \\
\text { models for exposure, one with VGDF and one with JEM, controlling for age, sex, race and smoking. Adjusted smoking risk } \\
\text { estimates are derived from the VGDF model; results for smoking were not substantively different in the JEM model. Results are } \\
\text { shown separately for the entire cohort and for the subset of subjects with GOLD stage II or greater, together with referents. }\end{array}$} \\
\hline
\end{tabular}

smoking-adjusted PAF of 24\%, which is midway between our two estimates of 13\% (VGDF) and 34\% (JEM). Moreover, our findings are well within the range of other reports using a variety of different approaches, providing critical evidence for the coherence of the association between occupation and COPD. ${ }^{1-6} 10$

We found that the joint exposure to smoking and occupational factors combined to increase the risk of COPD. This observation extends our earlier results in a different populationbased sample in which COPD was based on survey responses and not spirometry. ${ }^{8}$ Two other studies have also found interaction effects between smoking and occupational exposure for the risk of chronic bronchitis or chronic bronchitis plus airway obstruction (GOLD stage II or greater). ${ }^{9}{ }^{10}$ In the latter study, the combination of smoking and occupational exposure was associated with a nearly fivefold increase in the risk of COPD. ${ }^{9}$ Our findings further establish the joint risk of smoking and occupational exposures in a study that rigorously defined COPD based on a physician diagnosis of COPD, medical treatment for the condition and objective evidence of airway obstruction.

A key strength of our study is its recruitment of a large sample of patients with COPD with a broad spectrum of disease severity ranging from mild to severe, and the examination of matched referent subjects drawn from the same source population. Availability of pulmonary function testing on all subjects also enables more robust conclusions about how much COPD is attributable to occupational exposures.

Our study has several limitations. Misclassification of COPD cases could have occurred. Mitigating this limitation, all

Table 4 Joint associations between smoking and occupational exposure and COPD

\begin{tabular}{|c|c|c|c|c|c|}
\hline $\begin{array}{l}\text { Cigarette smoking status/ } \\
\text { occupational exposure }\end{array}$ & $\begin{array}{l}\text { Total number of } \\
\text { subjects (COPD + } \\
\text { referent) }\end{array}$ & $\begin{array}{l}\text { Probability of } \\
\text { COPD }\end{array}$ & $\begin{array}{l}\text { Excess } \\
\text { probability* }\end{array}$ & Crude OR & Adjusted OR \\
\hline \multicolumn{6}{|l|}{ Entire FLOW cohort } \\
\hline Never/yes & 145 & 0.60 & 0.16 & 1.92 (1.23 to 3.00$)$ & $1.98(1.26$ to 3.09$)$ \\
\hline Ever/no & 512 & 0.83 & 0.40 & 6.44 (4.42 to 9.39 ) & 6.71 (4.58 to 9.82 \\
\hline Ever/yes & 669 & 0.91 & 0.47 & 13.3 (8.90 to 19.8 ) & 14.1 (9.33 to 21.2 \\
\hline \multicolumn{6}{|l|}{ GOLD stage II or higher } \\
\hline Never/no & 136 & 0.26 & 0 & 1.0 & 1.0 \\
\hline Never/yes & 94 & 0.38 & 0.12 & 1.72 (0.98 to 3.03 ) & $1.69(0.96$ to 2.97$)$ \\
\hline Ever/no & 349 & 0.76 & 0.49 & 8.63 (5.49 to 13.6$)$ & 8.31 (5.27 to 13.1 ) \\
\hline Ever/yes & 465 & 0.87 & 0.61 & 19.1 (12.0 to 30.5 ) & $18.7(11.6$ to 30.0 \\
\hline
\end{tabular}

COPD, chronic obstructive pulmonary disease; FLOW, Function, Living, Outcomes and Work study; GOLD, Global Obstructive Lung Disease initiative.

*Difference in unadjusted probability of COPD compared with referent group (rounding error accounts for lack of agreement in some cells).

Adjusted odds ratio controls for age, sex and race in addition to smoking status. 
patients were required to have healthcare utilisation for COPD, a physician diagnosis of the condition and medical treatment of the disease. Moreover, we have previously demonstrated the validity of our approach using medical record review. ${ }^{14}$ In addition, the observed lifetime smoking prevalence in our COPD group was similar to that in other population-based epidemiological studies of COPD, further supporting the diagnosis of COPD rather than another condition. ${ }^{34} 35$ Finally, when we limited the definition of COPD to more severe disease based on spirometry (GOLD stage II or greater), the results were not substantively affected. We therefore have a high degree of confidence that our results are not significantly affected by misclassification bias.

Recruitment from a large health plan should also help to ensure that our results are applicable to patients who are treated for COPD in the general population. However, it is possible that the PAF calculated from members of a managed care organisation could differ from a general population-based sample. For example, if patients with occupationally-caused COPD left the workforce and consequently lost their health plan membership, our PAF estimate could actually underestimate the true occupational burden of COPD. Although the direction and magnitude of any bias cannot be fully quantified, we believe that it is likely to be small because KPMCP members are very similar to the regional population. ${ }^{12}$ Nonetheless, we acknowledge the potential pitfalls of generalising findings from a regional US study to other geographical locations.

Selection bias could have been introduced by non-participation in the study. There were some differences between subjects who did and did not participate in the interviews and clinic visits, but they were modest in scope and not likely to affect the relation between occupational exposure and COPD substantively. Nonetheless, we acknowledge the potential for selection bias as a limitation of our study.

Exposure misclassification was mitigated by our dual approach using independent exposure measures (VGDF and JEM). VGDF could be affected by either non-differential misclassification, which would bias toward the null, or differential misclassification if patients with COPD were more likely to remember and report VGDF exposure than those without COPD. ${ }^{36}$ The JEM is more likely to randomly misclassify exposure (ie, non-differential) and bias toward the null. In this sense, the PAF estimates derived from JEM and VGDF comprise a realistic range for the occupational contribution to COPD risk. Assessment of occupational exposure during the longest held job helped to ensure that exposure preceded the onset of COPD, even though we did not have detailed information about the temporal sequence of COPD incidence. ${ }^{37}$ Although we examined subjects with COPD and referent subjects at a single point in time, longitudinal follow-up of the cohort may yield additional exposure-response insights.

More than 20 years ago the relationship between dusty trades and COPD was highlighted by Becklake. ${ }^{38}$ Our study, taken together with the earlier literature, supports the likely causal nature of that association. Moreover, the findings indicate that smoking and occupation are powerful and interacting factors for developing COPD.

These results have shared relevance both for public health advocates who focus on primary prevention of the disease and clinicians who treat patients at risk for COPD and its progression. Public health efforts to prevent and treat COPD must target both smoking cessation and the reduction of adverse workplace exposures. Addressing one without the other will not effectively ameliorate the population burden of COPD.
Clinicians should be alert to high-risk occupational exposures in their patients. In patients employed in high-risk occupations, providers should consider targeted history taking, symptom assessment and spirometric evaluation in order to identify early stage disease and prevent its progression by reduction of relevant workplace exposures. ${ }^{39}$

\section{Funding: NHLBI/NIH R01HL077618.}

\section{Competing interests: None.}

Ethics approval: The study was approved by the University of California, San Francisco Committee on Human Research and the Kaiser Foundation Research Institute's institutional review board and all participants provided written informed consent.

\section{REFERENCES}

1. Balmes J, Becklake M, Blanc P, et al. American Thoracic Society Statement: Occupational contribution to the burden of airway disease. Am J Respir Crit Care Med 2003;167:787-97.

2. Blanc PD, Toren K. Occupation in chronic obstructive pulmonary disease and chronic bronchitis: an update. Int J Tuberc Lung Dis 2007;11:251-7.

3. Zock JP, Sunyer J, Kogevinas M, et al. Occupation, chronic bronchitis, and lung function in young adults. An international study. Am J Respir Crit Care Med 2001;163:1572-7

4. Hnizdo E, Sullivan PA, Bang KM, et al. Association between chronic obstructive pulmonary disease and employment by industry and occupation in the US population: a study of data from the Third National Health and Nutrition Examination Survey. Am J Epidemiol 2002;156:738-46.

5. Bergdahl IA, Toren K, Eriksson K, et al. Increased mortality in COPD among construction workers exposed to inorganic dust. Eur Respir J 2004;23:402-6.

6. Mak GK, Gould MK, Kuschner WG. Occupational inhalant exposure and respiratory disorders among never-smokers referred to a hospital pulmonary function laboratory. Am J Med Sci 2001;322:121-6.

7. Weinmann S, Vollmer WM, Breen V, et al. COPD and occupational exposures: a case-control study. J Occup Environ Med 2008;50:561-9.

8. Trupin L, Earnest G, San Pedro M, et al. The occupational burden of chronic obstructive pulmonary disease. Eur Respir J 2003;22:462-9.

9. de Meer G, Kerkhof M, Kromhout $H$, et al. Interaction of atopy and smoking on respiratory effects of occupational dust exposure: a general population-based study. Environ Health 2004;3:6.

10. Boggia B, Farinaro E, Grieco L, et al. Burden of smoking and occupational exposure on etiology of chronic obstructive pulmonary disease in workers of southern Italy. J Occup Environ Med 2008;50:366-70.

11. Karter AJ, Ferrara A, Liu JY, et al. Ethnic disparities in diabetic complications in an insured population. JAMA 2002;287:2519-27.

12. Krieger N. Overcoming the absence of socioeconomic data in medical records: validation and application of a census-based methodology. Am J Public Health 1992:82:703-10.

13. Eisner MD, Blanc PD, Sidney $\mathrm{S}$, et al. Body composition and functional limitation in COPD. Respir Res 2007;:8:7.

14. Sidney S, Sorel M, Quesenberry CP Jr, et al. COPD and incident cardiovascular disease hospitalizations and mortality: Kaiser Permanente Medical Care Program. Chest 2005;128:2068-75.

15. Calfee CS, Katz PP, Yelin EH, et al. The influence of perceived control of asthma on health outcomes. Chest 2006;130:1312-8.

16. Eisner MD, Yelin EH, Katz PP, et al. Risk factors for work disability in severe adult asthma. Am J Med 2006;119:884-91.

17. Pauwels RA, Buist AS, Calverley PM, et al. Global strategy for the diagnosis, management, and prevention of chronic obstructive pulmonary disease. NHLBI/WHO Global Initiative for Chronic Obstructive Lung Disease (GOLD) workshop summary. Am J Respir Crit Care Med 2001;163:1256-76.

18. Eisner MD, Yelin EH, Trupin L, et al. The influence of chronic respiratory conditions on health status and work disability. Am J Public Health 2002;92:1506-13.

19. Anon. Cigarette smoking among adults-United States, 1997. MMWR Morbidity and Mortality Weekly Report 1999;48:993-6.

20. US Bureau of the Census. Industry and occupation classification system Washington, DC: US Department of Commerces, 2000.

21. ECRHS Investigators. Protocol for the European Community Respiratory Health Survey. London: United Medical and Dental Schools of Guy's and St. Thomas' Hospitals, Department of Public Health Medicine, 1993.

22. Blanc PD, Ellbjar S, Janson C, et al. Asthma-related work disability in Sweden. The impact of workplace exposures. Am J Respir Crit Care Med 1999;160:2028-33.

23. Blanc PD, Eisner MD, Trupin L, et al. The association between occupational factors and adverse health outcomes in chronic obstructive pulmonary disease. Occup Environ Med 2004;61:661-7.

24. Blanc PD, Eisner MD, Balmes JR, et al. Exposure to vapors, gas, dust, or fumes: assessment by a single survey item compared to a detailed exposure battery and a job exposure matrix. Am J Ind Med 2005;48:110-7. 
25. Kennedy SM, Le Moual N, Choudat D, et al. Development of an asthma specific job exposure matrix and its application in the epidemiological study of genetics and environment in asthma (EGEA). Occup Environ Med 2000;57:635-41.

26. American Thoracic Society. Standardization of spirometry-1987 update. Statement of the American Thoracic Society. Am Rev Respir Dis 1987;136:1285-98.

27. American Thoracic Society. Standardization of spirometry, 1994 update. Am J Respir Crit Care Med 1995:152:1107-36.

28. Walters JA, Wood-Baker R, Walls J, et al. Stability of the EasyOne ultrasonic spirometer for use in general practice. Respirology 2006;11:306-10.

29. Perez-Padilla R, Vazquez-Garcia JC, Marquez MN, et al. The long-term stability of portable spirometers used in a multinational study of the prevalence of chronic obstructive pulmonary disease. Respir Care 2006:51:1167-71.

30. Hankinson JL, Odencrantz JR, Fedan KB. Spirometric reference values from a sample of the general US population. Am J Respir Crit Care Med 1999;159:179-87.

31. Fabbri LM, Hurd SS. Global strategy for the diagnosis, management and prevention of COPD: 2003 update. Eur Respir J 2003;22:1-2.

32. Greenland S, Drescher K. Maximum likelihood estimation of the attributable fraction from logistic models. Biometrics 1993:49:865-72.
33. Buist AS, McBurnie MA, Vollmer WM, et al. International variation in the prevalence of COPD (the BOLD Study): a population-based prevalence study. Lancet 2007;370:741-50.

34. Eisner MD, Balmes J, Katz PP, et al. Lifetime environmental tobacco smoke exposure and the risk of chronic obstructive pulmonary disease. Environ Health 2005; $4: 7$.

35. Mannino DM, Homa DM, Akinbami LJ, et al. Chronic obstructive pulmonary disease surveillance-United States, 1971-2000. MMWR Surveill Summ 2002:51:1-16.

36. Whitney E. Effects of misclassification of fume exposure. Am J Respir Crit Care Med 2008:177:1172; author reply 1172 .

37. Benke G, Sim MR, McKenzie DP, et al. Comparison of first, last, and longest-held jobs as surrogates for all jobs in estimating cumulative exposure in cross-sectional studies of work-related asthma. Ann Epidemiol 2008;18:23-7.

38. Becklake MR. Chronic airflow limitation: its relationship to work in dusty occupations. Chest 1985:88:608-17.

39. Harber P, Tashkin DP, Simmons M, et al. Effect of occupational exposures on decline of lung function in early chronic obstructive pulmonary disease. Am J Respir Crit Care Med 2007:176:994-1000.

\section{Pulmonary puzzle}

\section{An unusual cause of patchy ground-glass opacity}

\section{CLINICAL PRESENTATION}

In November 2006 a 33-year-old man was admitted with acute respiratory distress following a suicide attempt (50 hydroxyzine tablets and a vial of diazepam). The patient's medical history included a heroin and cocaine addiction which was substituted by methadone 4 years previously; epilepsy treated with valproic acid; and manic-depressive disorder treated with cyamemazine, olanzapine, zopiclone and diazepam. As a tobacco (15 pack-years) and cannabis smoker, the patient reported noticing that grit sand was mixed into a new batch of cannabis that he had changed to 2 weeks previously. He had smoked 5-6 non-filtered adulterated joints a day and complained of dyspnoea after each inhalation. A physical examination revealed dyspnoea with hypoxaemia at rest and diffuse rales,

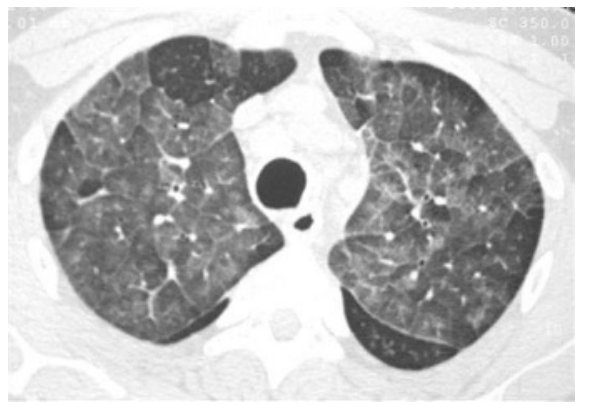

Figure 1 CT scan of the chest showing patchy areas of ground-glass opacities with interlobular septal thickening accentuated in both upper lung fields.

a high-pitched mid-inspiratory squeak and expiratory wheezing. The chest radiograph showed diffuse bilateral infiltrative shadows, mainly in the upper segments. No signs of clubbing, vasculitis or heart failure were identified. An axial thinsection CT scan revealed multiple patchy ground-glass opacities with accentuated interlobular septal thickening in both upper lung fields. Mediastinal lymphadenopathy was also observed (fig 1).

\section{QUESTION}

What is your diagnosis?

See page 74 for answer.

\section{N Just, ${ }^{1}$ J Delourme, ${ }^{1}$ C Delattre, ${ }^{2}$ A Liesse, ${ }^{3}$ F Steenhouwer ${ }^{1}$}

1 Department of Pneumology, Victor Provo Hospital, F59100 Roubaix, France; ${ }^{2}$ Department of Cytology, Lille University Hospital, F-59000 Lille, France; ${ }^{3}$ Department of Radiology, Victor Provo Hospital, F-59100 Roubaix, France

Correspondence to: Dr N Just, Department of Pneumology, Victor Provo Hospital, F-59100 Roubaix, France; nicolas.just@ch-roubaix.fr

Competing interests: None.

Thorax 2009;64:12. doi:10.1136/thx.2008.096875 